\title{
Vps41, a protein involved in lysosomal trafficking, interacts with caspase-8
}

\author{
Lu Wang ${ }^{1}$, Xiao Pan'1, Liangqiang He ${ }^{1}$, Rong Zhang1, Wei Chen ${ }^{1}$, Jing Zhang ${ }^{1}$, Min Lu ${ }^{1,2}$ and \\ Zi-Chun Hua ${ }^{1,2 \bowtie}$
}

1The State Key Laboratory of Pharmaceutical Biotechnology and School of Stomatology, Affiliated Stomatological Hospital, Nanjing University, Nanjing, PR China; ${ }^{2}$ Changzhou High-Tech Research Institute of Nanjing University, Changzhou, P. R. China.

\begin{abstract}
Caspase- 8 is a member of the cysteine-aspartic acid protease (caspase) family which plays a central role in apoptosis and development. We screened caspase- 8 interacting proteins from mouse T-cell lymphoma and 7.5-day embryo cDNA libraries by yeast two-hybrid system and obtained eleven positive clones, including Vacuolar protein sorting 41 (Vps41), a protein involved in trafficking of proteins from the late Golgi to the vacuole. The interaction of Vps41 with caspase-8 was confirmed by coimmunoprecipitation (co-IP) and co-localization studies in HEK293T cells. Co-IP experiments also showed that Vps41 binds to the p18 subunit of caspase- 8 through its WD40 region and RING-finger motif. Furthermore, we found that overexpression of Vps41 promotes Fasinduced apoptosis in A549 human lung adenocarcinoma cells. The cleavage of caspase-3, a caspase- 8 downstream effector, was increased when cells were transfected with Vps41-overexpressing plasmid. Together, these results suggest a novel interaction of caspase-8 with Vps41 and provide a potential role of Vps41 beyond lysosomal trafficking.
\end{abstract}

Key words: caspase-8, Vps41, yeast two-hybrid, protein interaction, apoptosis

Received: 25 June, 2012; revised: 24 October, 2012; accepted: 18 November, 2012; available on-line: 20 November, 2012

\section{INTRODUCTION}

Caspase- 8 was first described as a FADD-homologous interleukin $1 \beta$ converting enzyme (ICE)-like protease (Muzio et al., 1996), and plays a crucial role in apoptosis as an apical caspase. In FasL-induced apoptosis it is recruited to the cytoplasmic domain of Fas through the adaptor protein FADD. The proteolytic activity of the C-terminal domain of caspase- 8 appears after its cleavage initiated by the formation of the Fas-FADD-caspase- 8 complex. Caspase- 8 initiates the downstream apoptotic process through activating caspase-3 which cleaves PARP subsequently (Boldin et al., 1996; Cohen, 1997; Juo et al., 1998). In the mitochondrial apoptosis pathway caspase- 8 cleaves Bid, a pro-apoptotic member of the Bcl-2 family, which induces permeabilization of the outer mitochondrial membrane and helps mitochondria release cytochrome $c$, a crucial member of the apoptosome complex in mitochondrial apoptosis (Bossy et al., 1998; Li et al., 1998; Bratton et al., 2000; Fischer et al., 2003). Therefore, caspase- 8 mediates both death receptor-mediated apoptosis and intrinsic apoptosis.

VPS (vacuolar protein sorting) is a class of proteins originally identified in vesicular trafficking in yeast
(Radisky et al., 1997). Although more than 60 VPS genes have been identified, their functions in mammalian cells are only partly known.

Vps41 is a highly conserved protein. In yeast, it was indicated to be a subunit of both AP-3 (heterotetrameric adaptor protein complex 3) and HOPS (homotypic fusion and protein transport) complexes, both of which control protein trafficking from the late Golgi into the vacuole, an organelle analogous to the lysosome of mammalian cells (Conibear et al., 1998; Rehling et al., 1999; Wurmser et al., 2000; Brett et al., 2008; Cabrera et al., 2010). There are two isoforms of $\mathrm{hVps} 41$, both of which contain a WD40 domain, a CLH domain, as well as a RING-finger motif which mediates membrane association (McVey Ward et al., 2001). These two isoforms differ in that one of them lacks amino acids 83-107 of the amino terminus (Ruan et al., 2010). In mammalian cells Vps41 was found to be recruited by Arf-like GTPase Ar$18 \mathrm{~b}$ to lysosomes, and the two proteins then recruit other HOPS complex members to lysosomes. Vps41-silenced cells displayed a dramatic delay in delivery of proteins to lysosomes (Garg et al., 2011). These results indicate that the function of Vps41 in mammalian cells is similar to that in yeast, which is mediating the trafficking of proteins to vacuoles/lysosomes. Recent study has shown that Vps41 blocks downstream events in the apoptotic cascade including activation of caspase- 9 and caspase-3, and PARP cleavage in SH-SY5Y neuroblastoma cell line (Ruan et al., 2010). This result was then explained that Vps41 could enhance the clearance of misfolded and aggregated proteins, such as neurotoxic $\alpha$-synuclein (Harrington et al., 2012). Until present, all studies on Vps41 in mammalian cells have been based on its function of protein trafficking to lysosomes.

In this study, we investigated proteins capable of interacting with caspase- 8 by yeast two-hybrid screening and found Vps41 to be a novel caspase-8-interacting partner. We confirmed this interaction in mammalian cells. This result supplies a novel insight of apotential role of Vps41 beyond lysosomal trafficking.

\section{MATERIALS AND METHODS}

Cell culture and transfection. Human embryonic kidney HEK293 cells and human lung adenocarcinoma A549 cells were cultured in DMEM supplemented with

e-mail: zchua@nju.edu.cn

Abbreviations: FITC, fluorescein isothiocyanate; HOPS, homotypic fusion and protein transport; IP, immunoprecipitation; PBS, phosphate-buffered saline; PI, propidium iodide 
Table 1. Primer sequences used to generate truncated forms of hVps41 and hCaspase-8

\begin{tabular}{lll}
\hline Name & forward primer (5' to $\left.3^{\prime}\right)$ & reverse primer $\left(5^{\prime}\right.$ to $\left.3^{\prime}\right)$ \\
\hline WD40 & ttaaggatccatggcggaagcagtggag & ccgactcgagatgcttgtggatcaactg \\
CLH & ggtcggatccgattttgattcagagaaagc & cagtctcgagttaaacatgtgtgccaatgttg \\
RING & acacggatccgacccaattctactgattc & caatctcgagtcacatctccaaaattgcacttc \\
DED & cataggatccatggacttcagcagaaatc & aataaagctttcattcaaggctgctgcttctc \\
p18 & aattggatccaatggggaggagttgtgtg & ccggaagcttatcagtctcaacaggtatac \\
p10 & atatggatcctcagaggagcaaccctat & ccagaagctttcaatcagaagggaagac \\
\hline
\end{tabular}

$10 \%$ fetal bovine serum at $37^{\circ} \mathrm{C}$ in a humidified atmosphere containing $5 \% \mathrm{CO}_{2}$. Transient transfection of cells was performed using Lipofectamine 2000 (Invitrogen) according to the instructions provided by the manufacturer.

Yeast two-hybrid screening. Caspase- 8 was used as a bait in the yeast two-hybrid screening experiments. Bait gene encoding mCaspase- 8 was amplified by PCR and then cloned into the GAL4-DNA binding expression vector pGBKT7 DNA-BD (Clontech). The bait did not exhibit any intrinsic activation function in the yeast strain AH109 (Clontech). This bait was used to screen both mouse $\mathrm{T}$ lymphoma and 7.5-day embryo cDNA libraries constructed in the pACT2 DNA-AD vector (Clontech) and pretransformed into the AH109 strain. The transformants were then transformed with pACT2cDNA libraries and plated on yeast dropout medium lacking tryptophan (Trp), leucine (Leu), histidine (His) and adenosine (Ade). Colonies obtained on the dropout media (Trp-, Leu-, His- and Ade-) were checked for the activity of $\beta$-galactosidase according to the Clontech protocol, thereby selecting the plasmids encoding proteins capable of a two-hybrid interaction with caspase-8.

Plasmid constructs. The full-length hVps41 was amplified by PCR using the forward primer 5 '-att gga tcc atg gcg gaa gca gtg gag -3' and reverse primer 5'-ccg get cga gct att ttt tca tct cca aaa ttg c-3' from cDNA of HEK293T cells and cloned into the BamHI-XhoI sites of pRK5-Flag vector. The full length hCaspase- 8 was amplified using the forward primer 5 '-cat agg atc cat gga ctt cag cag aaa tc-3' and reverse primer 5'-cca gaa gct ttc aat cag aag gga aga c-3' by PCR from cDNA of HEK293T cells and cloned into the BamHI-Hindsites of pRK5-HA vector. Truncated forms of hVps41: WD40 (amino acids 1-546), CLH (amino acids 560-721)

Table 2. Proteins interacting with caspase-8 in yeast two-hybrid screen

\begin{tabular}{lll}
\hline No. & gene identified & NCBI accession number \\
\hline 2 & Mib2 (Mib2 mindbomb homolog 2) & NM_145124.2 \\
3 & Lztr1 (leucine-zipper-like transcriptional regulator) & NM_025808.3 \\
4 & Rpsa (ribosomal protein SA) & NM_011029.4 \\
6,8 & Eif3 (eukaryotic translation initiation factor 3) & NM_016876.3 \\
9 & Vps41 (vacuolar protein sorting 41) & NM_172120.4 \\
11 & Immt (inner membrane protein, mitochondrial) & NM_029673.2 \\
14,16 & Patz1 (POZ/BTB and AT hook containing zinc finger 1) & NM_019574.3 \\
28 & Guk1 (guanylate kinase 1) & NM_001159410.1 \\
29 & Nsun5 (NOL1/NOP2/Sun domain family, member 5) & NM_145414.2 \\
30 & Abhd16a (abhydrolase domain containing 16A) & NM_178592.3 \\
31 & Cryba4 (crystalline) & NM_021351.1 \\
\hline
\end{tabular}

and RING (amino acids 722-852) were cloned to pRK5-Flag vector, while truncated forms of hCaspase-8: DED (amino acids 1-190), p18 (amino acids 197374) and p10 (amino acids 375-479) were cloned to pRK5-HA vector, using appropriate primers (Table 1 ).

Co-immunoprecipitation. As many as $5 \times 10^{6}$ cells were lysed for $30 \mathrm{~min}$ on ice in lysis buffer $(20 \mathrm{mM}$ HEPES $\mathrm{pH}$ 7.4, $150 \mathrm{mM} \mathrm{NaCl}, 5 \mathrm{mM}$ EDTA, 10\% glycerol, $0.5 \%$ Triton X-100 and $10 \mu \mathrm{l} /$ $\mathrm{ml}$ protein inhibitor cocktail) after $48-\mathrm{h}$ transfection. The supernatant was incubated with $1 \mu \mathrm{g}$ anti-Flag (Sigma), antiHA (Sigma) or normal mouse immunoglobulin $G$ (IgG) (Millipore) antibody (IP antibody) at $4^{\circ} \mathrm{C}$ overnight. Thereafter incubation with $20 \mu \mathrm{l}$ protein $\mathrm{G}$ Sepharose beads (Upstate) was performed for $2 \mathrm{~h}$ at $4^{\circ} \mathrm{C}$. After washing three times with lysis buffer, the immunoprecipitates were incubated at $95^{\circ} \mathrm{C}$ for $5 \mathrm{~min}$ and probed with appropriate antibodies by western blotting.

Immunofluorescence. HEK293T cells were cultured on a cover slip and transfected with pRK5-Flag-hVps41 and pRK5-HA-hCaspase-8. Sixteen hours later, cells were fixed in 4\% paraformaldehyde in phosphate-buffered saline (PBS) at $4^{\circ} \mathrm{C}$ for $1 \mathrm{~h}$. After washing with PBS twice, cells were permeabilized in $0.5 \%$ TritonX-100 in PBS for $45 \mathrm{~min}$ at room temperature, and then washed twice with PBS. Subsequently, the cells were incubated with $3 \%$ BSA in PBS for $2 \mathrm{~h}$ to block nonspecific binding sites and thereafter incubated with appropriate antibodies diluted in $3 \%$ BSA in PBS at $4{ }^{\circ} \mathrm{C}$ overnight. Rabbit antiFlag (Cell Signal Technology), rabbit anti-HA (Sigma) and mouse anti-HA (Sigma) antibodies were diluted at 1:200, and anti-clathrin (Abcam) antibody was diluted at 1:75. After incubation the cells were washed three times with PBS on shaking table for $8 \mathrm{~min}$ each time, then incubated with Alexa Fluor 594 goat anti-mouse and Alexa Fluor 488 goat anti-rabbit antibodies (Invitrogen) diluted in $3 \% \mathrm{BSA}$ in PBS at $1: 1000$ for $1 \mathrm{~h}$ at room temperature in the dark. After washing three times, immunofluorescence was observed under a confocal laser scanning microscope.

Induction and analysis of apoptosis. A549 cells were cultured in a 24-wells plate, transfected with pRK5-Flag-hVps41 plasmid $0.5 \mu \mathrm{g} /$ well for $24 \mathrm{~h}$, and then treated with $200 \mathrm{ng} / \mathrm{ml}$ anti-Fas antibody (Millipore). After incubation for 6 or $24 \mathrm{~h}$, cells were collected, washed with binding buffer $(10 \mathrm{mM}$ HEPES $\mathrm{pH}$ 7.4, $140 \mathrm{mM} \mathrm{NaCl}$ and 2.5 $\mathrm{mM} \mathrm{CaCl}_{2}$ ) and stained with annexin $\mathrm{V}$-fluorescein isothiocyanate (FITC) in binding buffer for $10 \mathrm{~min}$ on ice, then propidium iodide (PI) was added and cells were analyzed immediately on a FACSCalibur flow cytometer using CellQuest software. Cells in the early stage of apoptosis were stained positive for annexin V-FITC and negative for PI, while those in the late stage of apoptosis were stained positive for both annexin V-FITC and PI. 


\section{RESULTS}

\section{Eleven clones were obtained through yeast two-hybrid screening}

To identify proteins that interact with caspase-8, cDNA libraries derived from mouse $\mathrm{T}$ lymphoma and 7.5-day embryo were screened using a yeast two-hybrid system with the bait plasmid pGBKT7-mCaspase-8 which expresses the bait protein caspase-8. Thirty-one candidates were obtained by the screening. We cloned these 31 DNA fragments to pACT2 vector and retransformed them into the caspase- 8 bait yeast strain AH109 independently to confirm their interactions with caspase- 8 in yeast by two-hybrid system and finally ob-

a

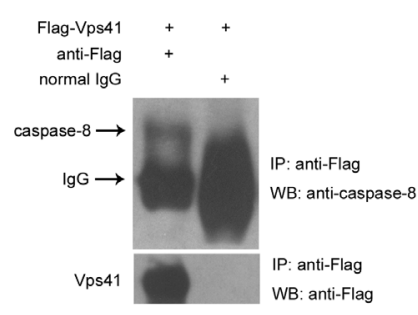

b
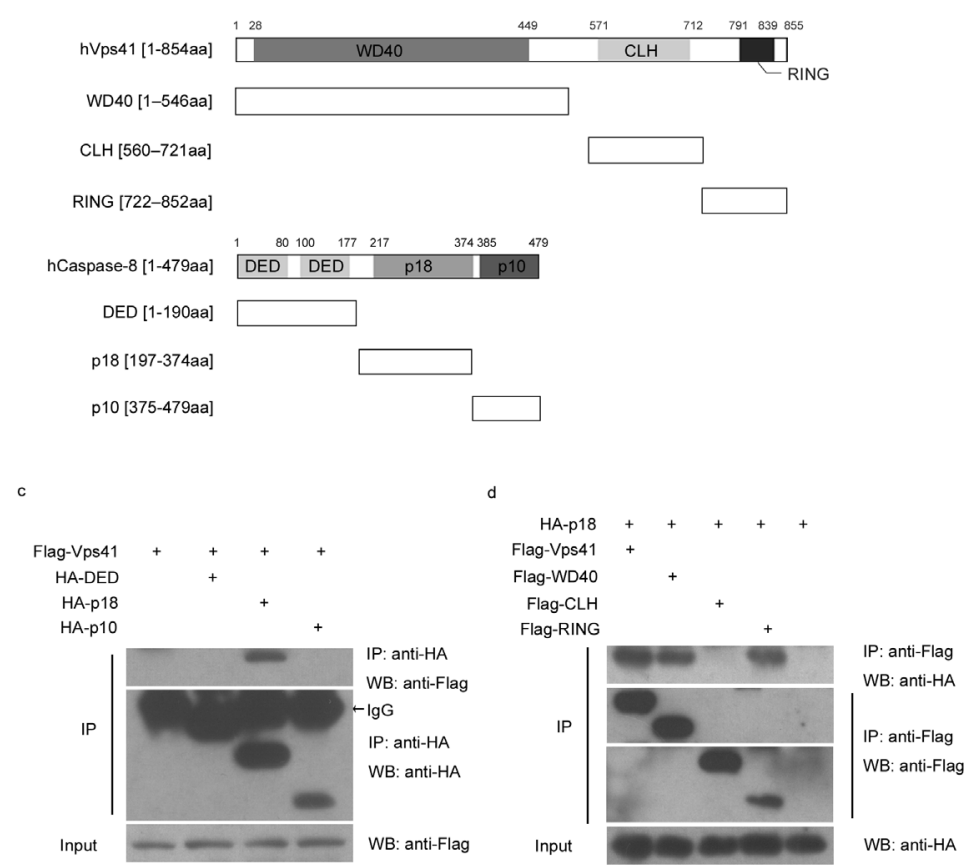

Figure 1. Vps41 interacts with caspase-8 in HEK293T cells.

(a) Flag-Vps41 was transfected into HEK293T cells. Twenty-four hours later, cells were lysed and protein complexes were co-immunoprecipitated by either anti-Flag antibody or normal lgG. Caspase-8 and Vps41 were dectected by western blotting with anti-caspase-8 and anti-Flag antibodies, respectively. (b) Structural information of hVps41 and hCaspase-8 and their truncated forms cloned into the expression vector and used in this study. (c) Flag-Vps41 was co-transfected with pRK5-HA vector or pRK5-HA-DED, pRK5-HA-p18, pRK5-HA-p10 into HEK293T cells, co-IP was performed with anti-HA antibody, then Vps41 was detected with anti-Flag antibody and the domains of caspase- 8 were detected with anti-HA antibody by western blotting. The input of Vps41 is also shown at the bottom. (d) HA-p18 was co-transfected with pRK5-Flag-Vps41, pRK5-Flag-WD40, pRK5-Flag-CLH, pRK5-Flag-RING or pRK5-Flag vector into HEK293T cells. Co-IP was performed with anti-Flag antibody, then the p18 subunit was detected with anti-HA antibody, and full-length Vps41 or its domains were detected with anti-Flag antibody. The input of p18 is shown at the bottom. tained 11 positive clones. These clones were sequenced, and blast searches were performed against GenBank to ( transcriptional regulator), Vps41 (vacuolar protein sorting 41), and Guk1 (guanylate kinase 1) (Ta-

\section{Vps41 binds to caspase-8 in HEK293T cells}

Eleven proteins interacting with caspase- 8 were identified in yeast two-hybrid screen. To confirm their interaction with caspase- 8 in mammalian cells, we cloned these After a 48-h transfection with the plasmids encoding putative interacting proteins, HEK293T cells were lysed and immunoprecipitated with either anti-Flag antibody or normal mouse immunoglobulin $G$ as negative control. The co-immunoprecipitates were subsequently immunoblotted with anti-caspase- 8 antibody (Calbiochem). For the cells transfected with pRK5-Flag-hVps41, endogenous caspase- 8 was detected in the co-immunoprecipitates (Fig. 1a, upper panel), and expression of the recombinant Flag-hVps41 was detected with antiFlag antibody (Fig. 1a, lower panel). This result demonstrates that Vps41, a protein involved in lysosomal trafficking, binds to endogenous caspase- 8 in HEK293T cells.

In order to determine which domains play crucial roles in the interaction of Vps41 with caspase-8, a series of plasmids were constructed. The WD40, CLH and RING domain of Vps41 were cloned individually into pRK5-Flag vector, whereas DED, p18 and p10 domain of caspase- 8 were cloned into pRK5-HA vector according to the structural information of these two proteins provided by Swiss-prot (Fig. 1b).

The pRK5-Flag-hVps41 plasmid was co-transfected with pRK5-HA-DED, pRK5-HA-p18, pRK5-HA-p10 or pRK5HA vector in HEK293T cells, and cell lysates were subjected to immunoprecipitation with anti-HA antibody. CoIP results revealed that the p18 subunit of caspase- 8 , but not the DED or p10 domain, interacts with Vps41 (Fig. 1c). We further focused on the domains of Vps41 that may interact with caspase-8. In yeast, the N-terminus of Vps41 is required for binding to adaptor protein complex AP-3, an important complex involved in trafficking of proteins from Golgi and endosomal compartments to both the vacuole/lysosome and the plasma membrane, whereas the C-terminal CLH domain directs homo-oligomerization of Vps41 (Ruan et al., 2010). The RING-finger motif has been shown to mediate membrane association in mammalian cells (Bossy et al., 1998). The pRK5-HA-p18 plasmid was co-transfected with pRK5-Flag-hVps41, pRK5-FlagWD40, pRK5-Flag-CLH, pRK5-Flag- 

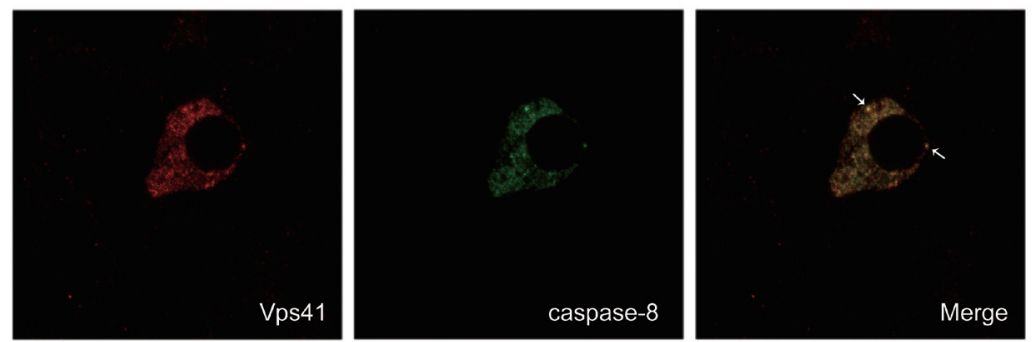

b
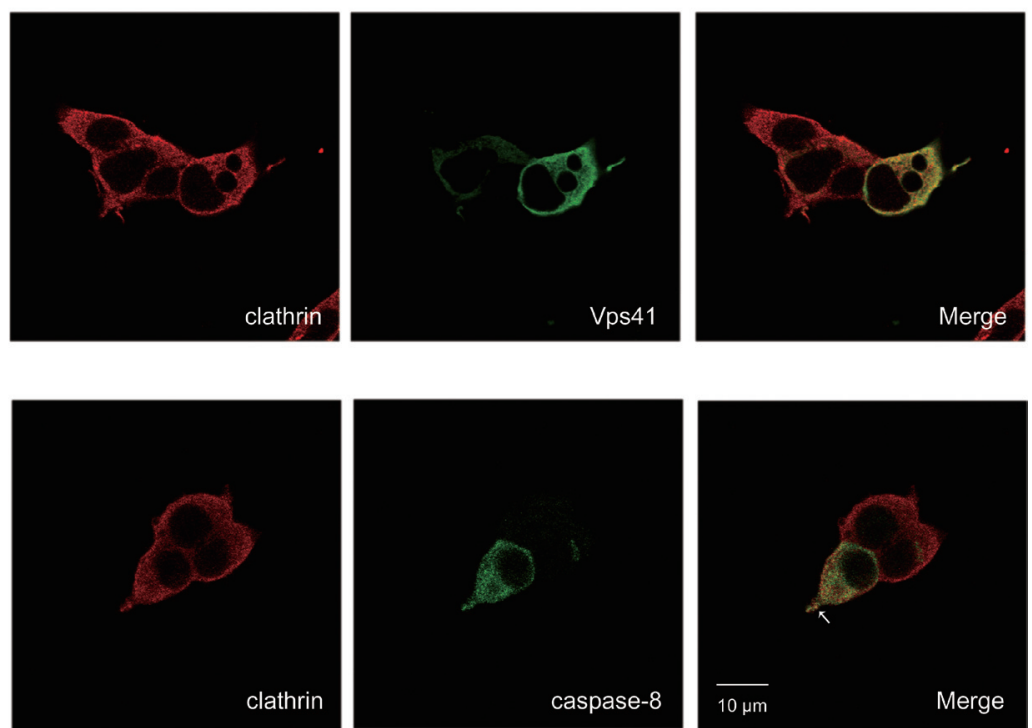

Figure 2. Vps41 and caspase-8 co-localize with clathrin in HEK293T cells.

HEK293T cells were transfected with pRK5-Flag-hVps41 and pRK5-HA-hCaspase-8. Cells were fixed after $16 \mathrm{~h}$ and incubated with (a) mouse anti-Flag and rabbit anti-HA antibodies (b) mouse anti-clathrin and rabbit anti-Flag antibodies (c) mouse anti-clathrin and rabbit ant-HA antibodies at $4^{\circ} \mathrm{C}$ overnight, followed by staining with Alexa Fluor 594 goat anti-mouse and Alexa Fluor 488 goat anti-rabbit antibodies. Co-localization is shown in the right panel.

a

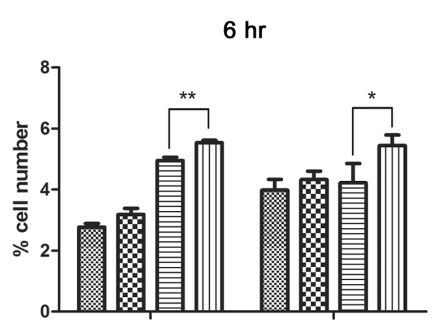

early apoptosis
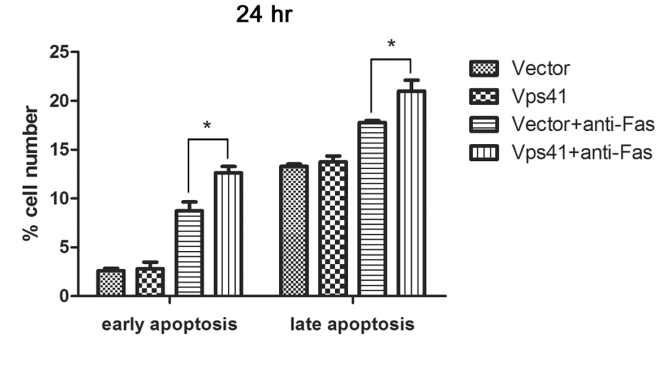

b

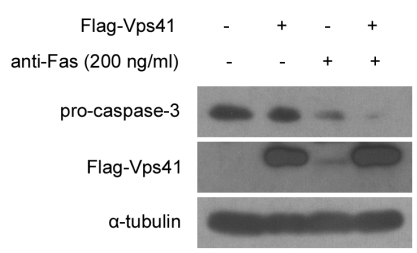

Figure 3. Vps41 promotes apoptosis induced by anti-Fas antibody in A549 cells.

A549 cells were transfected with vector or pRK5-Flag-Vps41. (a) Twenty-four hours later, cells were treated or not with $200 \mathrm{ng} / \mathrm{ml}$ anti-Fas antibody in 5\% FBS culture medium for 6 or $24 \mathrm{~h}$ and apoptosis was analyzed by double staining with annexin $\mathrm{V}$ and PI (values are mean \pm S.D., ${ }^{*} p<0.05,{ }^{*} p<0.01$, Student's $t$-test). (b) Twenty-four hours later, cells were treated or not with $200 \mathrm{ng} / \mathrm{ml}$ anti-Fas antibody in 5\% FBS culture medium for $24 \mathrm{~h}$. Pro-caspase-3 was detected by western blotting with anti-caspase-3 antibody.
RING or pRK5-Flag control vector in HEK293T cells, followed by immunoprecipitation with anti-Flag antibody. Co-IP results showed that when cells were transfected with plasmids encoding WD40, RING or fulllength Vps41, HA-p18 was detected in the co-immunoprecipitates by immunoblotting (Fig. 1d). These observations reveal that Vps41 binds to the p18 subunit of caspase- 8 through both its WD40 region and the RING-finger motif.

\section{Vps41 co-localizes with caspase-8 in HEK293T cells}

To determine the cellular localization of Vps41 interacting with caspase-8, confocal microscopy was performed on HEK293T cells expressing Flag-hVps41 and HA-hCaspase- 8 simultaneously. The result demonstrated co-localization of these two proteins in the cytoplasm (Fig. 2a). Since Vps41 is believed to cover transport vesicles and help the docking onto lysosomes, we were interested in whether caspase- 8 also localizes to vesicles. We examined the localization of Vps41 and caspase- 8 to determine whether they co-localize with the vesicle marker clathrin. Immunofluorescent co-localization assay revealed that Vps41 is co-localized with clathrin and caspase- 8 is partially co-localized with clathrin (Fig. 2b, 2c), which indicates that Vps41 may interact with caspase-8 on vesicles in HEK293T cells.

\section{Vps41 promotes apoptosis induced by anti-Fas antibody in A549 cells}

To investigate whether Vps41 plays a role in caspase8-mediated apoptosis, we chose a Fas-sensitive cell line A549 to perform apoptosis induction and analysis since caspase- 8 is known as an apical caspase in Fas-induced apoptosis. We transfected A549 cells with $\mathrm{hVps} 41$ and treated the cells with anti-Fas antibody for 6 or $24 \mathrm{~h}$ to induce apoptosis. Flow cytometric data revealed that among the A549 cells transfected with Vps41, early apoptotic cells (annexin $\mathrm{V}+/ \mathrm{PI}-)$ were more fragment $(5.54 \pm 0.07 \%)$ compared to the control $(4.95 \pm 0.11 \%) \quad(n=3$, 
$p<0.01$ ) and late apoptotic cells (annexin $\mathrm{V}+/ \mathrm{PI}+$ ) were also more fragment $(5.44 \pm 0.35 \%)$ compared to the control cells $(4.22 \pm 0.63 \%)(n=3, p<0.05)$ after a 6-h anti-Fas treatment. Also after 24-h anti-Fas treatment early apoptotic cells were more abundant among those transfected with Vps41 $(12.63 \pm 0.65 \%)$ compared to the control $(8.75 \pm 0.91 \%)(n=3, p<0.05)$, as were late apoptotic cells were increased $(21.00 \pm 1.11 \%)$ vs. $(17.79 \pm 0.18 \%) \quad(n=3, p<0.05)$ (Fig. 3a). We also estimated the protein level of pro-caspase-3 after anti-Fas treatment for $24 \mathrm{~h}$ and found pro-caspase-3 reduced in cells transfected with Vps41 compared to control cells transfected with vector (Fig. 3b). As caspase-3 is a downstream effector of caspase-8, these results show that Vps41 may promote apoptosis and this promotion is probably through regulating caspase- 8 activity in A549 cells.

\section{DISCUSSION}

In previous studies, $\mathrm{Vps} 41$ was shown to control protein trafficking. It covers transport vesicles and helps the dock onto lysosomes. In this study we first validated a direct interaction of Vps41 with caspase-8, a pro-apoptosis protease. Co-IP experiments showed that the p18 domain, which is known as a subunit of activated caspase- 8 , interacts with Vps41. However, as the cleavage reaction by caspase- 8 is an instanteneous process that is difficult to be detected by co-IP, and we did not find any cleavage band of Vps41 in apoptotic cells induced by anti-Fas antibody (unpublished data), we believe that Vps41 is not a substrate of caspase-8.

In recent studies, over-expression of $h V p s 41$ was shown to enhance the clearance of misfolded and aggregated $\alpha$-synuclein, and to block downstream events in the apoptotic cascade including activation of caspase- 9 and caspase- 3 , and PARP cleavage in SH-SY5Y neuroblastoma cells. Vps41 was supposed to be a useful target for developing therapeutic strategies for human Parkinson's disease (Ruan et al., 2010; Garg et al., 2011). However, our experiments showed that in the A549 human lung adenocarcinoma cell line, Vps41 appeared to have a promoting effect on Fas-induced apoptosis. This result may have some relationship with the direct interaction of Vps41 with caspase-8, and is also consistent with the observation that a Vps41 gene mutation which resulted in premature termination of protein synthesis was found in gastric cancer $(1 / 30)$ and colorectal cancer $(1 / 40)$, while DNA from normal tissues from the same patients showed no evidence of the mutation (An et al., 2012). The latter results indicate that a defect of this protein may be associated with tumorigenesis itself or with apoptosis residence of tumor cells. Correspondingly, another research showed that Vps41 gene was significantly amplified in 12 of $13(92 \%)$ primary effusion lymphoma (PEL) cell lines based on Affymetrix arrays (Roy et al., 2011). Together with the finding mentioned previously that overexpression of Vps41 protected SH-SY5Y cells from apoptosis, these results indicate that Vps41 may play different roles in different tissues. The function or effect of Vps41 in human diseases requires further research. Our result that Vps41 interacts directly with caspase-8 supplies a novel insight of a potential role of Vps41 beyond lysosomal trafficking and suggests a possible mechanism of Vps41 participation in the regulation of caspase-8-mediated apoptosis.

\section{Acknowledgments}

This study was supported in part by grants from the National Key Basic Research Project from the Chinese Ministry of Science and Technology (2012CB967004), the Chinese National Natural Sciences Foundation (50973046, 31200572, 31070706, 81121062, 31071196), the Jiangsu Provincial Natural Science Foundation (BZ2010074, BZ2011048, BK2011228, BK2011573, BZ2012050), and the Bureau of Science and Technology of Changzhou (CZ20100008, CE20115034, CZ20110028, CJ20115006).

\section{REFERENCES}

An CH, Kim YR, Kim HS, Kim SS, Yoo NJ, Lee SH (2012) Frameshift mutations of vacuolar protein sorting genes in gastric and colorectal cancers with microsatellite instability. Hum Pathol 43: 40-47.

Boldin MP, Goncharov TM, Goltsev YV, Wallach D (1996) Involvement of MACH, a novel MORT1/FADD-interacting protease, in Fas/APO-1 - and TNF receptor-induced cell death. Cell 85: 803815.

Bossy-Wetzel E, Newmeyer DD, Green DR (1998) Mitochondrial cytochrome $c$ release in apoptosis occurs upstream of DEVD-specific caspase activation and independently of mitochondrial transmembrane depolarization. EMBO J 17: 37-49.

Bratton SB, MacFarlane M, Cain K, Cohen GM (2000) Protein complexes activate distinct caspase cascades in death receptor and stress-induced apoptosis. Exp Cell Res 256: 27-33.

Brett CL, Plemel RL, Lobingier BT, Vignali M, Fields S, Merz AJ (2008) Efficient termination of vacuolar Rab GTPase signaling requires coordinated action by a GAP and a protein kinase. I Cell Biol 182: 1141-1151.

Cabrera M, Langemeyer L, Mari M, Rethmeier R, Orban I, Perz A, Bröcker C, Griffith J, Klose D, Steinhoff HJ, Reggiori F, Engelbrecht-Vandré S, Ungermann C (2010) Phosphorylation of a membrane curvature-sensing motif switches function of the HOPS subunit Vps41 in membrane tethering. J Cell Biol 191: 845-859.

Cohen GM (1997) Caspases: the executioners of apoptosis. Biochem J 326: 1-16

Conibear E, Stevens TH (1998) Multiple sorting pathways between the late Golgi and the vacuole in yeast. Biochim Biophys Acta 1404: 211-230.

Fischer B, Coelho D, Dufour P, Bergerat JP, Denis JM, Gueulette J, Bischoff P (2003) Caspase 8-mediated cleavage of the pro-apoptotic BCL-2 family member BID in p53-dependent apoptosis. Biochem Biophys Res Commun 306: 516-522.

Garg S, Sharma M, Ung C, Tuli A, Barral DC, Hava DL, Veerapen N, Besra GS, Hacohen N, Brenner MB (2011) Lysosomal trafficking, antigen presentation, and microbial killing are controlled by the Arflike GTPase Arl8b. Immunity 35: 182-193.

Harrington AJ, Yacoubian TA, Slone SR, Caldwell KA, Caldwell GA (2012) Functional analysis of VPS41-mediated neuroprotection in Caenorhabditis elegans and mammalian models of Parkinson's disease. J Neurosci 32: 2142-2153.

Juo P, Kuo CJ, Yuan J, Blenis J (1998) Essential requirement for caspase-8/FLICE in the initiation of the Fas-induced apoptotic cascade. Curr Biol 8: 1001-1008.

Li H, Zhu H, Xu CJ, Yuan J (1998) Cleavage of BID by caspase 8 mediates the mitochondrial damage in the Fas pathway of apoptosis. Cell 94: 491-501.

McVey Ward D, Radisky D, Scullion MA, Tuttle MS, Vaughn M, Kaplan J (2001) hVPS41 Is Expressed in Multiple Isoforms and Can Associate with Vesicles through a RING-H2 Finger Motif. Exp Cell Res 267: 126-134.

Mellman I, Warren G (2000) The road taken: past and future foundations of membrane traffic. Cell 100: 99-112.

Muzio M, Chinnaiyan AM, Kischkel FC, O'Rourke K, Shevchenko A, Ni J, Scaffidi C, Bretz JD, Zhang M, Gentz R, Mann M, Krammer PH, Peter ME Dixit VM (1996) FLICE, a novel FADD-homologous ICE/CED-3-like protease, is recruited to the CD95 (Fas/ APO-1) death-inducing signaling complex. Cell 85: 817-827.

Radisky DC, Snyder WB, Emr SD, Kaplan J (1997) Characterization of VPS41, a gene required for vacuolar trafficking and high-affinity iron transport in yeast. Proc Natl Acad Sci USA 94: 5662-5666.

Rehling P, Darsow T, Katzmann DJ, Emr SD (1999) Formation of AP-3 transport intermediates requires Vps41 function. Nat Cell Biol 1: $346-353$.

Roy D, Sin SH, Damania B, Dittmer DP (2011) Tumor suppressor genes FHIT and WWOX are deleted in primary effusion lymphoma (PEL) cell lines. Blood 118: 32-39. 
Ruan Q, Harrington AJ, Caldwell KA, Caldwell GA, Standaert DG (2010) VPS41, a protein involved in lysosomal trafficking, is protective in Caenorhabditis elegans and mammalian cellular models of Parkinson's disease. Neurobiol Dis 37: 330-338.
Wurmser AE, Sato TK, Emr SD (2000) New component of the vacuolar class $\mathrm{C}-\mathrm{V}$ ps complex couples nucleotide exchange on the $\mathrm{Ypt} 7$ GTPase to SNARE-dependent docking and fusion. J Cell Biol 151: $551-562$. 